\title{
CALCULATION OF PARTICLE MOTIONS AT THE HEAD AND TAIL OF A BUNCH FOR THE UNIVERSITY OF MARYLAND ELECTRON RING *
}

\author{
$\underline{\text { Y. Li }}{ }^{\#}$, R. Kishek, M. Reiser and J.G. Wang ${ }^{\mathrm{a}}$, \\ Institute for Plasma Research, University of Maryland, College Park, MD 20742
}

\begin{abstract}
The end effect in a bunched beam is caused by the space-charge forces, which accelerate particles at the bunch head and decelerate particles at the bunch tail. It occurs in high-current linear accelerators and rings [1]. In the University of Maryland Electron Ring (UMER) project $[2,3,4]$, the energy of particles at the very ends of a rectangular bunch is $15 \%$ different from that of the main part of the bunch. Assuming a negligible transverse space charge effect at the edge of the bunch, one dimensional single particle calculation is performed by a matrix formalism [6] to estimate the ratio of particle loss due to the end effect. Two possible cases are examined: with no induction gap, and with three induction gaps. If three induction modules are used, for a small number of turns, the particle loss is less than $0.15 \%$, which is small enough to be acceptable. Simulation by code CIRCE [8] is in progress.
\end{abstract}

\section{INTRODUCTION}

A compact electron ring, UMER, $[2,3,4]$ is designed and being developed at the University of Maryland for transport of a low-energy, highly space-change dominated beam in a circular lattice. A $10 \mathrm{keV}, 100 \mathrm{~mA}$, $50 \mathrm{~ns}$ electron beam is injected into the ring from the injector. The ring has 36 dipoles and 36 FODO focusing periods to keep the beam along the designed orbit.

When a bunch with a rectangular current profile is transported, the strong longitudinal space charge force causes an edge erosion and large energy spread [5]. Three induction gaps are used to restore the pulse shape. The energy of the bunch edge varies periodically due to the longitudinal expansion and restoration. In UMER, the maximum energy difference between the edge and the main part of the bunch is as large as $1.5 \mathrm{keV}$, which is $15 \%$ of the beam energy. This large energy difference leads to a serious deviation from the desired beam orbit when these edge particles go through a circular lattice with dispersion. Also, the focusing function of quadrupoles, which depends on the particle energy, will be different to the edge particles. Therefore, the head and tail particles have different behaviour and may be lost.

For a rectangular bunch with a uniform velocity distribution, when the beam length is much larger than

"Work supported by the US Department of Energy.

"Email: liyun@glue.umd.edu

${ }^{a}$ : Present Address: ORNL and Visiting at BNL. the beam radius, the variation of the line charge density $\lambda$ and relative velocity $v_{r}$ can be found in [5]. The typical erosion process and formation of head and tail are shown in Figure 1, where $C_{\mathrm{s}}=\sqrt{\left(e g I_{0}\right) /\left(4 \pi \varepsilon_{0} m v_{0} \gamma^{5}\right)}$ is the space charge wave velocity, with $v_{0}$ the beam center velocity.

Before reaching the "cusp" point, the expanded bunch can be restored by an "ear field" produced by induction gaps. After the bunch is contracted back into a rectangular shape, it will repeat the expansion. Thus, the energy of the head particles vary periodically from 1.5 $\mathrm{keV}$ higher than the beam to $1.5 \mathrm{keV}$ lower than the beam, with the same time-average value as particles in the main part of the beam.

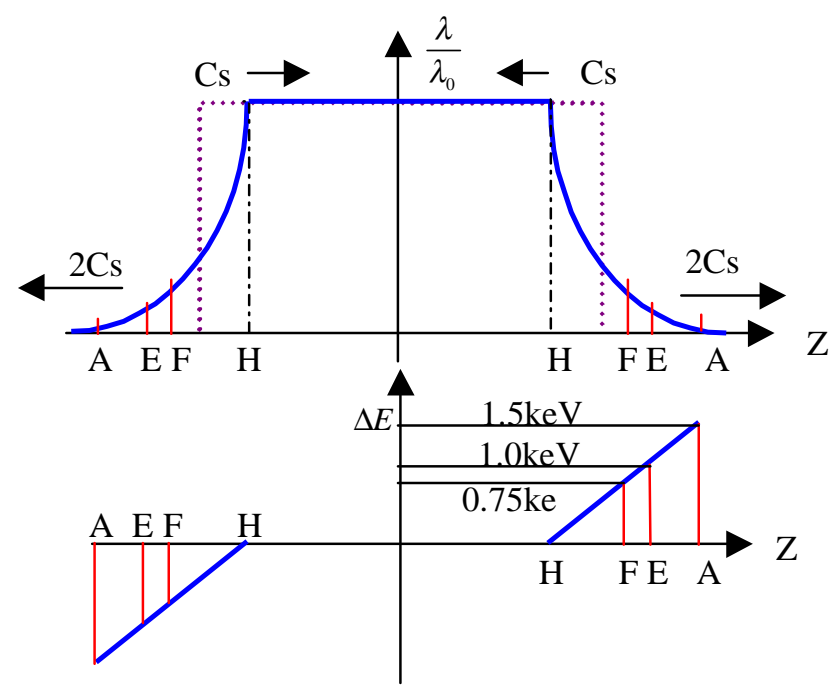

Figure 1: Bunch expansion process.

\section{MODEL USED IN CALCULATION}

In this paper, a simple one-dimensional model is developed to estimate the ratio of particle loss due to the end effect. It is supposed that the transverse space charge effect can be neglected due to the relatively small line charge density at the edge of the bunch. And the longitudinal space charge only changes the energy of particles, and therefore, the focus function of quadrupoles and the bending radius in the dipole. The particle motion is tracked by the matrix formalism [6].

The hard edge approximation is made for the quadrupoles and dipoles. The focusing function of the quadrupole is determined by the energy of the particle at the time it passes through the quadrupole. The particle gets a kick in the horizontal direction when it goes 
through the dipole; the strength of the kick depends on the particle energy. A MATLAB program is written to calculate the particle trajectory by using the matrix formalism.

The particles whose oscillation amplitude is larger than $20 \mathrm{~mm}$ are considered lost, and whose amplitude is less than $20 \mathrm{~mm}$ will remain in the beam pipe. By varying the initial condition $\left(x, x^{\prime}\right)$ of the particle at some longitudinal location $\mathrm{z}$ and observing the maximum deviation, the stable area in the phase space at the location $\mathrm{z}$ for that particle is obtained. After comparing the stable area with the matched beam distribution in the phase space, the ratio of particle loss at the position $\mathrm{z}$ is known. Several particles were picked up in calculation according to their longitudinal positions in the bunch. These particles are marked in Figure 1. They are: head A, head E, tail A, tail E, and tail F.

There are three induction gaps in one revolution and 12 FODO periods between two adjacent induction gaps. One period is defined as from the middle of the long drift section to the middle of the next long drift section. The matrix elements are as following [6]:

- Drift space:

$\left(\begin{array}{c}x \\ x^{\prime}\end{array}\right)_{\text {out }}=\left[\begin{array}{cc}1 & S \\ 0 & 1\end{array}\right] \cdot\left(\begin{array}{l}x \\ x^{\prime}\end{array}\right)_{\text {in }}$,

- Quadrupole:

$\left(\begin{array}{l}x \\ x^{\prime}\end{array}\right)_{\text {out }}=\left[\begin{array}{cc}\cos k l & \frac{\sin k l}{k} \\ -k \sin k l & \cos k l\end{array}\right] \cdot\left(\begin{array}{l}x \\ x^{\prime}\end{array}\right)_{\text {in }}$ for focusing

or $\left(\begin{array}{c}x \\ x^{\prime}\end{array}\right)_{\text {out }}=\left[\begin{array}{cc}\cosh k l & \frac{\sinh k l}{k} \\ k \sinh k l & \cosh k l\end{array}\right] \cdot\left(\begin{array}{l}x \\ x^{\prime}\end{array}\right)_{\text {in }}$ for defocusing

With $\mathrm{k}^{2}=\frac{B^{\prime} q}{m_{0} c \beta \gamma}$.

- Dipole:

$\left(\begin{array}{l}x \\ x^{\prime}\end{array}\right)_{\text {out }} \approx\left[\begin{array}{cc}1 & \rho \sin \theta \\ 0 & 1\end{array}\right] \cdot\left(\begin{array}{l}x \\ x^{\prime}\end{array}\right)_{\text {in }}+\left(\begin{array}{c}\rho(1-\cos \theta) \\ \theta\end{array}\right) \cdot \delta$

Where $\delta=\frac{\Delta P}{P}$ is the relative momentum error.

\section{CALCULATION RESULTS}

The matched beam distribution in phase space is obtained by TRACE-2D [7]. It is further supposed that the beam is uniformly distributed inside the ellipse of the matched beam distribution in phase space.

The acceptance is obtained by sending an ideal particle with initial condition $\left(x, x^{\prime}\right)$ into the FODO lattice, and observing its maximum amplitude of betatron oscillation. The initial conditions $\left(x, x^{\prime}\right)$ which corresponding to maximum amplitude of $20 \mathrm{~mm}$ compose the acceptance ellipse. For $10 \mathrm{keV}$ ideal particle, the acceptance is shown in Figure 2 (the large ellipse) with the matched beam distribution (the small ellipse).

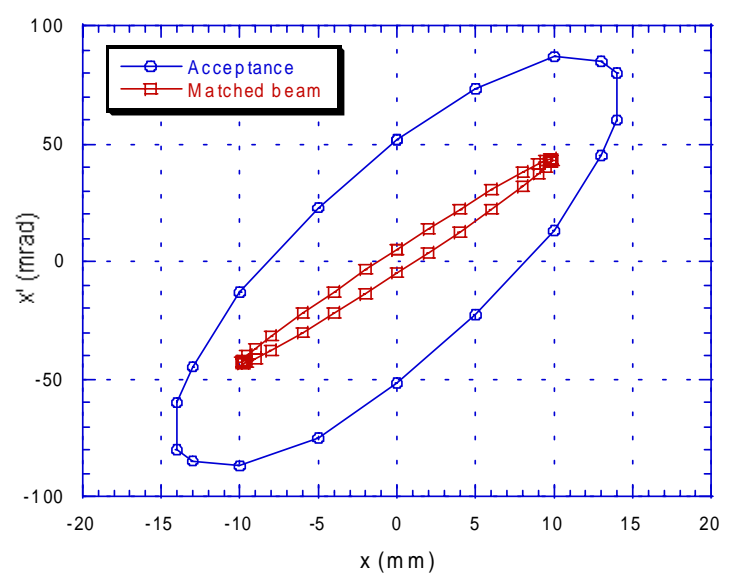

Figure 2: Matched beam distribution and the acceptance.

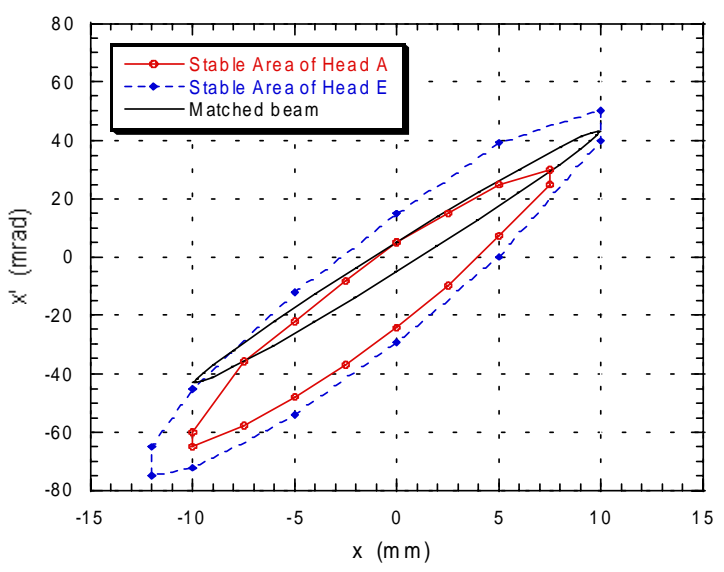

Figure 3: Stable Area of Head A and Head E, with 3 gaps.

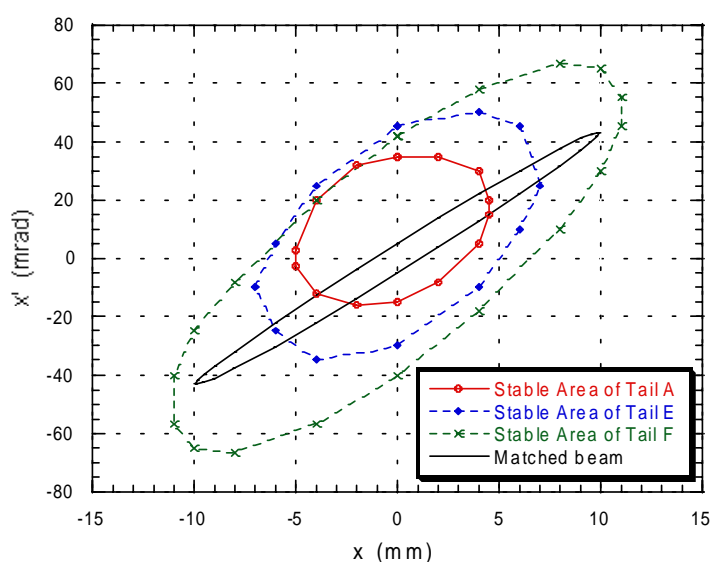

Figure 4: Stable area of tail A, tail E and tail F, with 3 gaps.

In order to know the ratio of the particle loss, the stable area is compared with the matched beam 
distribution. The stable area of the head particles and tail particles are shown in Figure 3 and Figure 4. The ellipses are corresponding to the initial condition $\left(x, x^{\prime}\right)$ that causes $20 \mathrm{~mm}$ deviation; so the particles outside of the stable area ellipse will be lost.

From Figure 3, the ratio of particle loss for particle head $\mathrm{A}$ and head $\mathrm{E}$ are 0.4 and 0 . So the ratio of particle loss in bunch head is less than

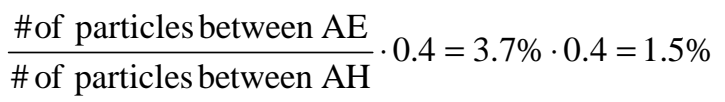

From Figure 4, the ratio of particle loss for particle tail $\mathrm{A}$, tail $\mathrm{E}$, and tail $\mathrm{F}$ are respectively $0.5,0.3$ and 0 . So the ratio of particle loss in bunch tail is less than

$$
\begin{aligned}
& \frac{\# \text { of particles between } \mathrm{AE}}{\# \text { of particles between } \mathrm{AH}} \cdot 0.5+\frac{\# \text { of particles between } \mathrm{EF}}{\# \text { of particles between } \mathrm{AH}} \cdot 0.3 \\
& =3.7 \% \cdot 0.5+(12.5 \%-3.7 \%) \cdot 0.3=4.5 \%
\end{aligned}
$$

There is $5 \%$ of particles located in the head and tail. So, the total ratio of particle loss is less than $5 \% \times 4.5 \% \div 2+5 \% \times 1.5 \% \div 2=0.15 \%$

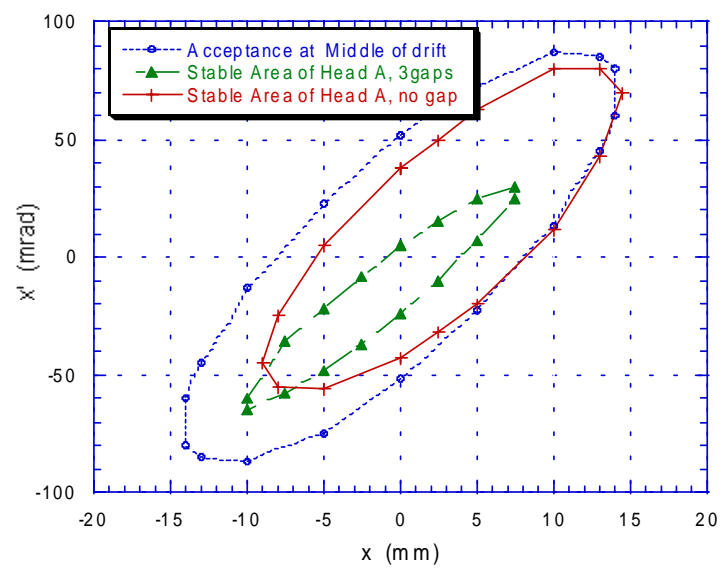

Figure 5: Comparison of stable area of particle head A in 2 cases: with 3 gaps and with no gap.

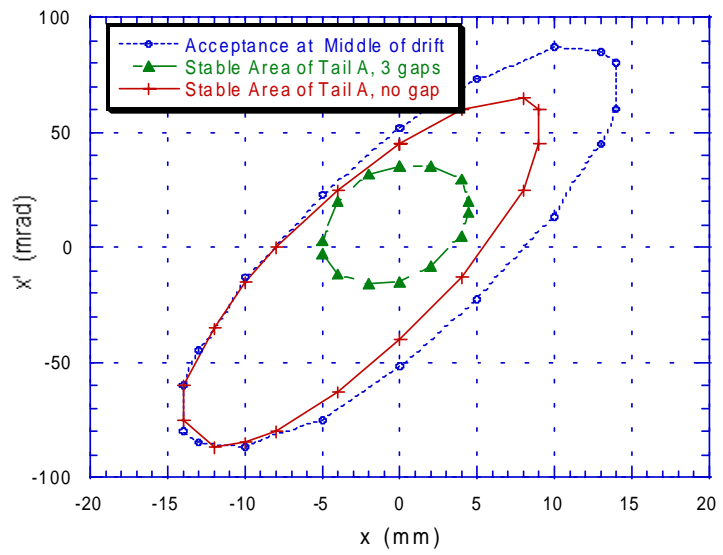

Figure 6: Comparison of stable area of particle tail A in 2 cases: with 3 gaps and with no gap.
In some experiments when we care more about beam position, the induction gaps will be changed into resistive beam position monitors, resulting in no periodic energy variation. In Figure 5 and Figure 6, the stable area in this case is compared with that of three induction gaps. From the comparison we know that the stable area is larger if there is no induction gap. The ratio of loss is smaller compared to the case with three induction gaps. However, this model only applies to the first three turns before the "cusp" point. After that the model is no longer correct.

Simulation by code CIRCE [8] is going on. But there is no final result yet. The first author would like to acknowledge Bill Sharp of LLNL for his great help in using this code.

\section{SUMMARY}

The calculation results show that the end effect is not of serious concern in UMER. In the case of no induction gaps, for the first three turns considered here, the particle loss rate is negligibly small. In the case of three induction gaps, less than $0.15 \%$ particles will be lost for a small number of turns. This number is small enough so that no special arrangement is needed to overcome this effect during the initial operation of UMER.

\section{REFERENCES}

[1] D. A. Edwards and M. J. Syphers, "An Introduction to the Physics of High Energy Accelerators", Wiley, New York, 1992.

[2] M. Reiser, et al., "The Maryland Electron Ring for Investigating Space-Charge Dominated Beams in a Circular FODO System,"in these proceedings.

[3] J.G. Wang, S. Bernal, P. Chin, R. A. Kishek, Y. Li, M. Reiser, M. Venturini, Y.Zou, T. Godlove, I. Haber, and R. C. York, Nucl. Instrum. Methods Phys. Res. A. (1998), vol. 415, no. 1-2, p.422-7.

[4] http://www.ipr.umd.edu/ebte/ring.

[5] D.X. Wang, J.G. Wang, and M. Reiser, "Restoration of Rectangular Pulse shape after Edge Erosion for a Space-Charge Dominated Electron Bunch”, Phys. Rev. Lett. 73(1), 66 (1994).

[6] M. Reiser, "Theory and Design of Charged Particle Beams", Wiley, New York, 1994.

[7] K. R. Crandall and D. P. Rusthoi, "TRACE 3-D Documentation", LA-UR-90-4146, Los Alamos National Laboratory .

[8] Bill Sharp, private communications. 